\title{
A Neural Dynamics and Oscillator Interference Mixed Model of the Grid Cells for Spatial Recognition
}

\author{
Naigong $\mathrm{Yu}^{*}$, Ziwei Luo and Yujia Zhai \\ Beijing University of Technology, Beijing, China \\ ${ }^{*}$ Corresponding author
}

\begin{abstract}
Grid cells in the MEC (media entorhinal cortex) of rats show repetitive hexagon firing pattern while moving through the environment. In the deeper layer of the MEC, a kind of interneurons have both the head-directing (HD) and grid-like firing properties. The predecessors' observations indicate the grid cells have ubiquitous intracellular rhythmic oscillating. Moreover, the grid cells' firing frequencies have interesting approximate proportion relationship with the animal's moving velocity. However, animal's perception of the motor speed might be affected by many other factors such as visual signals or external information. The oscillation interference process inevitably follows with error accumulating. Based on what we have mentioned above, we put forward an improved grid cell model that handle speed signal in a plausible way and ameliorate the effects of phase noise. And this paper's model is achieved by combining two prevailing theories together which might be closer to the scientific truth of grid cells.
\end{abstract}

Keywords-Grid Cells; OI; CAN; Speed Perception; Phase Reset.

\section{INTRODUCTION}

Since 1971 that O'Keefe and Dostrovsky discovered the place cell in the hippocampal CA1, an entirely new branch of neuroscience has emerged [1]. The place cells that fire specifically according to animal's locations give rise to a new speculation about the spatial recognition [2]. During the past 40 years, more and more scientists devote into this new field, especially the discovering of Grid cells led the research to a new height [3]. Grid cells are neurons distributed in the MEC, and they have stable spatially specific firing activities like place cells . Furthermore, their repeatedly regularly distributed firing fields form hexagonal patterns which draw an extreme interest in the scientific community. Nowadays grid cells have caused great enthusiasmin the academic circles but the characteris tics of grid cells have not been thoroughly investigated [4]. With the traditional navigation largely relying on landmarks, these spatial specific firing neurons supply us a new view that specific signals could represent space using information generated by an animal's movements [5]. Certainly, there are heterogeneous populations of cells in the field of hippocampal. Furthermore, in the deeper layer a kind of interconnect cells are found that have both the HD characteristic and the grid-like characteristic. Even the grid cells in different layers have completely different directional characteristics.

O'Keefe's cognitive map theory emphasizes an important process called Path integration (PI), supposing upper the place cells existing dead reckoning computing about the moving vector [6]. The hexagon repeatedly firing grid cells in some degree coincide with the precisely vector computing, consequently arising a univers al attitude that grid cells are the PI executor. Behavioral and neurophysiological studies indicate that grid cells can combine the HD signal from the HD cells and other distance information to keep track of animal's relative position [5].

Modeling parts of the brain function is a challenging job. Since the discovering of the grid cells, scientists attempt to propose theoretic approaches to mimic the information processing power. There are two dominant perspectives to account for the generation of the grid cells. One view is by the firing rates of the neurons, versus another by the synchrony among neural oscillators. Continuous attractor network (CAN) is based on the neural dynamics theory [7]. In CAN models, the attractor state is achieved by the symmetrical recurrent interactions between grid cells to express the cells. And the connection strength of the grid cells is defined by the animal's movement velocity. The activity bumps shifting along relative dynamics trajectories displays the PI process. Electrophysiological (EEG) evidence showed the intracellular activities have obvious oscillating at theta frequency and the extracellular oscillating appearing at a different frequency. Oscillatory Interference(OI) model derives from the discovering of the phase precession which existing in the place cells [8]. They proposed that the summation of the two oscillations result in the neuron's firing activities [9]. The short of the OI repetitive outcomes exactly apposite to peculiar grid cells' regular pattern. The frequency of oscillations is normally modulated by the speed of the rat, then the firing rate and phase will depend on the location of animals. The OI model has obvious deficiency that the grid pattern gradually degrade with the phase noise accumulating.

Although the typical CAN model and OI model base on different theories to explain path integration, they theoretically account for complementary properties of grid cell. However, these two models are not mutually exclusive theories, and there have been a lot of attempts to combine continuous attractor and os cillatory interference networks together [10]. A CAN and OI joint model might better interpret the spatial cognitive cell's phenomenon and essence. 


\section{MATERIALS}

\section{A. Modules}

The whole model is based on the complicated transmission circuit about HD cells, conjunctive cells, grid cells and place cells as shown in the Figure 1.

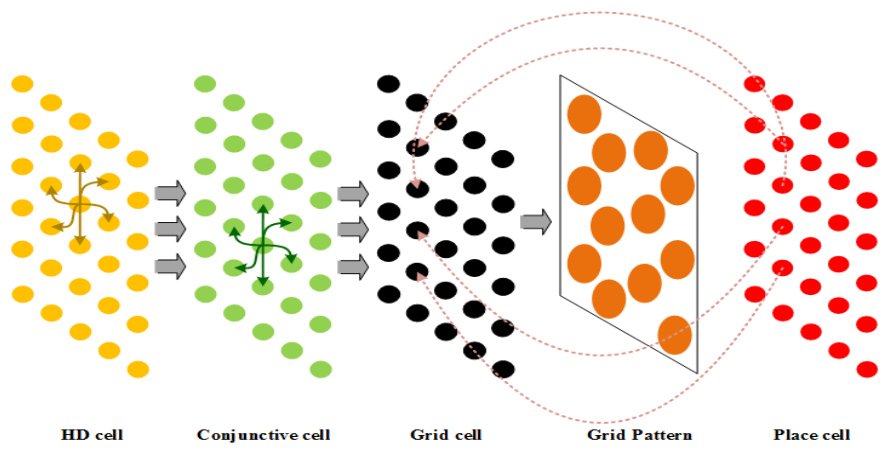

FIGURE I. THE ENTIRE GRID CELL MODEL

The yellow balls are HD cells that have different preferred directions. The HD cells are implemented by the normal CAN model which is vividly displayed in Figure 2. The arrow in each ball symbolizes that HD cell's preferred direction in which that $\mathrm{HD}$ cell has strongest firing activity. And the abundant HD cells could cover omni-direction. The green balls represent the conjunctive cells which evince favorable HD property through the directly input from the attractor network achieved by plenty of HD cells. What's more these conjunctive cells perform significant interconnections to behave considerable grid-like feature. Despite sharing the common preferred direction, the VCOs in the same ring circuit represent different phases covering the whole cycle time. Then the amount of conjunctive circuits embracing the total $360^{\circ}$ form a crucial part of the grid cells (Figure 3 ). The pivotalblack balls are grid cells that receive inputs from both the preceding cells and downstreamplace cells. In the familiar ring circuit, the deep colored circles stand for different oscillatory rhythms. In the network, the cells' interconnection and temporal phase changing result in a 'activity bump' with dynamical stability and the 'activity bump' shifts around the circuit according to the animal moves through space. The grid cell activity is assumed as a stable network state by recurrent inhibitory connectivity between conjunctive cells and feedforward input from place cells. Several conjunctive cells ring circuits with diverse preferred directions are integrated as a crucial part the grid cell's input. As a consequence, the regular hexagon performance is achieved through an interference between theta-band oscillations in grid cells and other inputs of them.

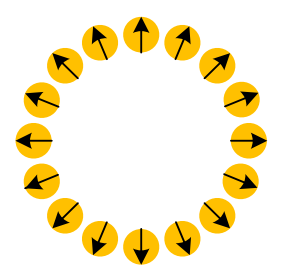

FIGURE II. A PLAIN ATTRACTOR CIRCLE FOR HD CELLS

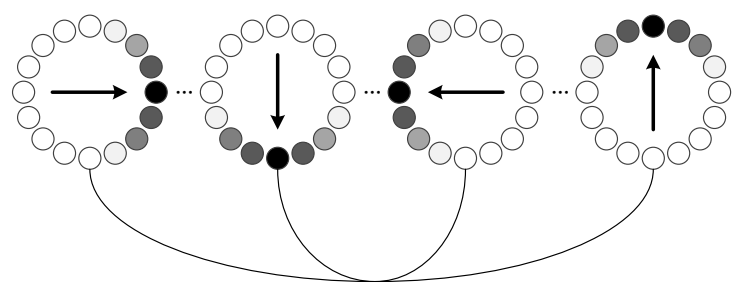

FIGURE III. CONJUNCTIVE CELL MODEL

\section{B. The Perception System of the Motor Velocity}

Since O'Keefe introduced the suspected speed cell firstly in 1998, he proposed a conjecture that speed is directly encoded in the animal body according to a sketchy observation result about the firing rate of speed cell and speed of running on a linear track [11].

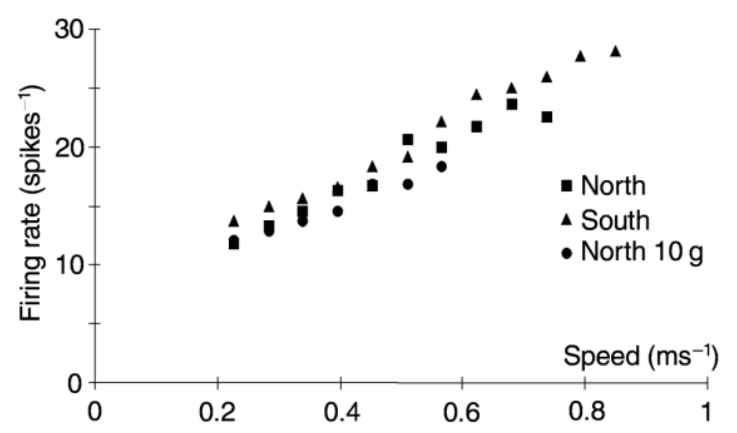

FIGURE IV. O'KEEFE'S OBSERVATION OF THE FIRING RATE AND THE RUNNING SPEED [11]

Nowadays many researchers use the firing rate to represent the speed. There is a widespread view that the firing rate is proportional to the speed. Recently Moser's research has further confirmed the existence of speed cells and defined the speed cell's standard [12]. The speed cells are particular population of MEC neurons, distinctive from the normal population of spatial specific firing cells, like grid cells, HD cells, place cells. There are approximately merely $15 \%$ of the MEC neurons showing a positive, linear response to running speed, and low levels of spatial and directional information [12]. The rare speed cells (46) can precisely code a speed signal which is independent with the visual inputs. However, how the original precise code come from is still unknown. The strategy to get the accurate speed-rate relationship of each individual has no generalization. To enable efficient path integration, the speed signal fits two essential, linear speed-rate relationship, and contextual invariance. When the animals move through the world, the information coming from multiple source integrate the perception of its own motion. As a result, there are inevitable deviations between the perceived speed and the actual speed. The pas sive motion experiment also indicates that Rat's perception of their velocity deviates from the actual speed in the space. The speed information used directly in spatial recognizing process is a synergistic code which is encoded by synergetic speed relative signals. 


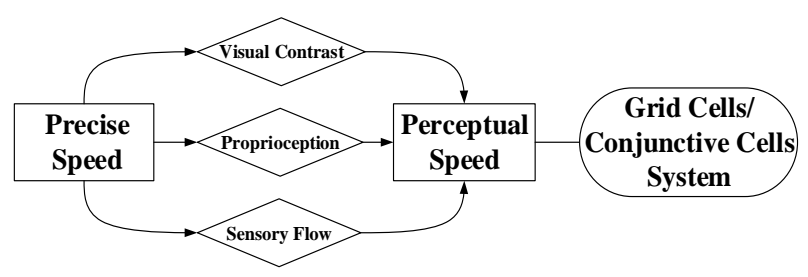

FIGURE V. THE PERCEPTION SYSTEM OF THE MOT OR VELOCITY

As shown in Figure 5, the original precise speed coded by the speed cells is transferred to the effective perceptual speed which is directly working in the conjunctive cells and grid cells system. The transition has three main influencing factors, visual contrast, proprioception and vestibular system inputs. In the same environment, the visual contrast might produce deflection of the distance in the visual cortex. The proprioception arising from muscles and joints provides the animal with valuable motion cues. As the important balance device, the vestibular system affords the body with rotating and linear acceleration information. And the equalizing process adjust the speed signal coded precisely by the speed cells to another accommodating the organism habits. All the three main factors can be generalized to a perceptualextent. In this paper, we creatively introduce a new coefficient perception factor to well interpret the role of grid cells. This variable reflects all the possible influences of the speed perception considering the moving conditions. When the animals enter a new fixed environment, the factor is a random, and progressively increase to a steady value through the environment exploration. If the environment is dynamical changed, the perception factor will change according to the real environment. In the whole internal spatial recognizing system, we logically mimic the conjunctive cells like the oscillators controlled by the final perceptualspeed.

\section{The Error Rectification}

As described before (Figure 3), in this improved model, we assume with several oscillations arranged in a ring attractor circuit sharing a common preferred direction to mimic the conjunctive cell. According to the relationship between velocity and the neuron firing rate, we assume them as velocitycontrolled oscillators (VCOs). For the prevalent errors, we mainly adopt two measures to make the error rectification in both active and passive. That is to couple the VCOs in each ring circuit and reset the VCOs' phase configuration in each theta cycle.

In the normal OI model, the oscillating property customarily entail the subsequent instability of firing pattern. The inevitable phase noise accumulating in each VCO input culminates the grid firing patterns increasingly dispersing and incoherent, even finally degrading. Previous theoretical work has demonstrated that what matters is the relative phase between VCOs works rather than the absolute phases [13]. We argue that the system should obey profitable phase changing in unison, but prevent other phase differences. To maintain the relative phases relationship, we arrange VCOs in ring attractors circuit and establish coupling links between VCOs to keep the internal consistency of VCOs (e.g. Figure 6). Based on aforesaid conjunctive cell model, we put forward the VCOs coupling whose detailed conceptual model is presented in fig6. Afterward the chained VCOs' ring attractor arraying allows all the phase changing synchronously.

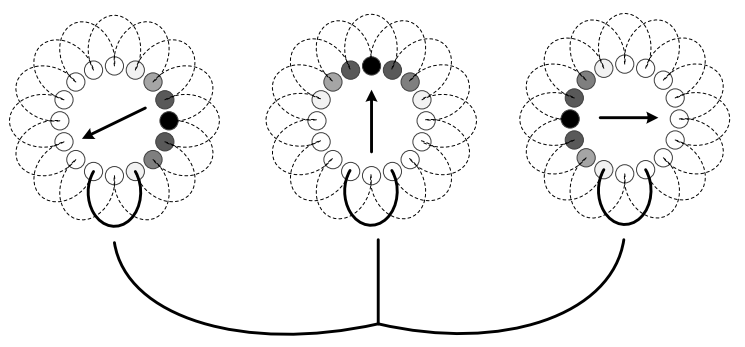

FIGURE VI. THE CONCEPTUAL MODEL OF THE VCOS COUPLING

Considering the situation that errors in each VCO ring attractor circuit, we take a step setting repeated reanchoring actions also called phase resetting. The grid cell firing activity conversely inclines the attractor circuit to shift toward the direction conducing grid cells activity. The unsupervised Hebb learning could execute the mutual projections between grid cells and VCO conjunctive cells circuits. And then mutual information conducts the activity bump's attractor dynamic that stay at a relatively constant position in the circuit. In our simulation, we random initialize the phase trains in each ring circuit and the Hebb learning always tend to promote the connecting weight vary towards generating grid pattern. Phase resetting occurs at the end of per theta cycle.

The error correction measures during the model's phase noise accumulating exactly coincide with the recurrent inhibition events between the neurons. The coupling process increase the model's computational complexity, then facilitate the model greater synchronous encoding capability and error tolerance. Conversely the grid cells' coupling behavior also support the neural synchrony during space recognizing process. Ultimately the VCOs coupling and the phase resetting could effectively diminish the noise in the model and ensure the gridlike firing pattern steady.

\section{MATHEMATICAL FORMATION}

According to the potential analysis, we mathematically describe the neurons in a differential equation like Equation 1:

$$
\mathrm{C} \frac{\mathrm{d}}{\mathrm{dt}} \mathrm{u}(t)=-\frac{1}{\mathrm{R}}\left(\mathrm{u}(\mathrm{t})-\mathrm{u}_{0}\right)+\mathrm{i}(\mathrm{t})
$$

$(C)$ is the membrane capacitance, and $(R)$ is the membrane leakage resistance, $(\mathrm{u}(\mathrm{t}))$ is the membrane potential, $(\mathrm{i}(\mathrm{t}))$ is the input current, $\left(\mathrm{u}_{0}\right)$ is the decreased current when $(\mathrm{i}(\mathrm{t}))$ decrease to zero. Spikes are fired whenever the membrane potential $(\mathrm{u}(\mathrm{t}))$ reaches a threshold $\left(\mathrm{u}_{\mathrm{t}}\right)$, after which it is reset to $\left(\mathrm{u}_{\text {reset }}\right)$. As shown in the fig In each ring attractor circuit, the initial VCOs phase series is $\left(\varphi_{\mathrm{VCO}}\right)=\left(\varphi_{1}, \varphi_{2}, \varphi_{3}, \ldots, \varphi_{\mathrm{n}}\right)$, and all the VCOs share a unitary preferred direction $\left(\phi_{\mathrm{VCO}}\right)$. We equippart the whole $360^{\circ}$ direction into six chunks, then each ring circuit has a certain preferred direction. To better analog the realistic membrane dynamics, we postulate a plenty of cells in each circuit, and within the circuit the phase offset is evenly distributed. 
Constitute the relationship between motor speed and the burst-firing frequency in equation 2 :

$$
\begin{gathered}
v_{V C O}(t)=v_{P S}(t) \cos \left(\emptyset(t)-\emptyset_{V C O}\right) \\
f_{V C O}(t)=f_{\text {base }}+\alpha v_{V C O}(t)
\end{gathered}
$$

( $\left.f_{b a s e}\right)$ is the baseline frequency, and the $\left(v_{P S}(t)\right)$ is the animals perceptual speed, $\alpha$ is a constant. $\left(\mathrm{v}_{\mathrm{VCO}}\right)$ denote the component of movement velocity along the VCO's preferred direction $(\phi)$. As for perceptualspeed, we express it in equation 3 according to this paper's argument.

The phase resetting carried out in every theta circle follows the equation 4.

$$
\begin{gathered}
\overrightarrow{\varphi_{\mathrm{R}}}=\arg \left(\frac{1}{\mathrm{~N}} \sum_{\mathrm{j}=1}^{\mathrm{N}} \mathrm{n}_{\mathrm{j}} \mathrm{e}^{\left(\overrightarrow{\varphi_{\mathrm{j}}^{*}}\right)}\right) \\
\overrightarrow{\varphi_{\mathrm{VCO}}}=\overrightarrow{\varphi_{\mathrm{VCO}}}-\alpha_{\mathrm{VCO}}\left(\overrightarrow{\varphi_{\mathrm{VCO}}}-\overrightarrow{\varphi_{\mathrm{R}}}\right)
\end{gathered}
$$

In the phase offset train $\left(\varphi_{\mathrm{j}}\right), \varphi_{\mathrm{j}}$ parallel to peak firing in each cell $\mathrm{j},\left(\varphi_{\mathrm{R}}\right)$ is assumed as the mean of the whole cells in the ring, $\left(n_{j}\right)$ is the number of the spikes fired in the theta cycle.

\section{THE EXPERIMENT AL RESULTS AND ANALYSIS}

The experience data is real membrane voltage traces having been analyzed in terms of "ramp" depolarization and theta modulation which is published by Barry in 2007 including 20 mins' rats randomly foraging in a $1 \mathrm{~m}^{2}$ arena (in a uniform scalar velocity regardless the moving direction). We use the valuable data to test the improved model mentioned above. The experience results are shown below.
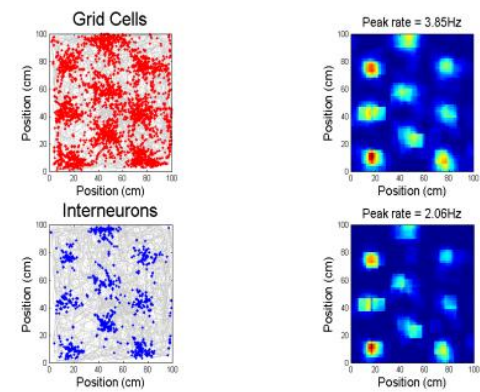

\section{FIGURE VII. THE CAN AND OI MIXED MODEL}

As shown in Figure 7, our model succes sfully achieved a grid cell firing rate map forming the grid like firing pattern. The gray lines express the trajectory of the rat, the red and blue spots stand for the cells' firing activity. This stable activity grids proved our model's effectiveness in modeling the spatial specificity firing, namely, this model indeed built a reliable space information encoding hierarchy.

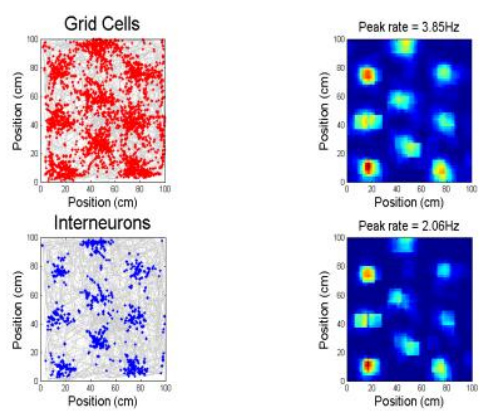

\section{FIGURE VIII. GRID CELLS' FIRING ACTIVITY IN FAMILIAR ENVIRONMENT}
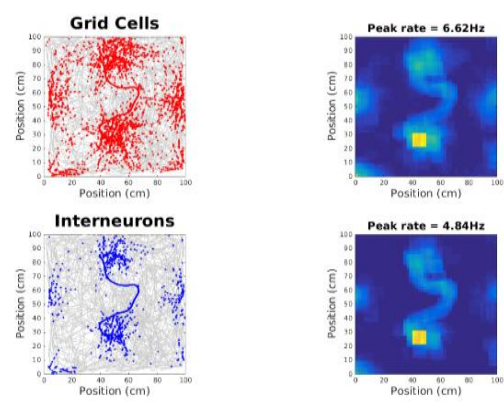

\section{FIGURE IX. GRID CELLS' FIRING ACTIVITY IN NOVEL ENVIRONMENT}

The proposed perceptual speed system worked well in this grid cell model (Figure 8\&9). As shown in Figure 8, in a familiar environment, grid cells behaved great grid-like firing characteristic and stability. In this situation, besides the uniform excitatory input and recurrent inhibition, the stable perceptual speed ensure that the network produces a coherent grid firing pattern in a location determined by the random initial VCO phases and phase resetting then acts to align VCO phases with that grid firing pattern. However, when the rat entered novel environment (Figure 9), the grid cells' firing field expanded obviously. Under the strict variable control, the difference could only be the perceptual speed. This phenomenon implicitly verified our perception system of the motor velocity. The same moving velocities result in great difference in the grid pattem stability.

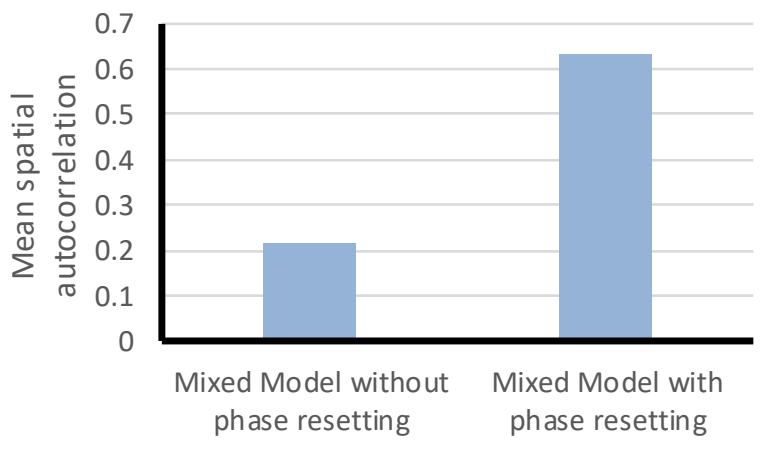

\section{FIGURE X. MEAN SPATIAL AUTOCORRELATION COMPARISON}

As for the error rectification strategies, we used the spatial autocorrelation to evaluate the stability of the grid pattern which 
can conversely reflect the influence of the error [14]. Figure 10 showed the spatial and relative stability of grid firing pattems with phase noise. Our CAN and OI mixed model features in substantial coupling between and in ring circuits. The mean spatial autocorrelation of the mixed model with phase resetting is obviously higher than same model without phase resetting. The experiment result demonstrates the phase resetting's effectiveness.

\section{SUMMARY}

This paper successfully proposes an improved grid cell model that can account for more properties about the grid cells than before. The most accurate might not be the most appropriate. The finding of the speed cell undisputedly reveals significant mystery of brain spatial cognitive. Nonetheless, with the perception factor involved, the creative speed information treatment well explains the grid pattern expand firstly while the animal entry a novel environment. The mammal's spatial cognizing process results from multiple information integrating and complicated network connection. And this paper's model is achieved by combining two prevailing theories together which might be closer to the scientific truth of grid cells. The successful performance of the CAN and OI mixed model additionally prove the superiority of the model. Under the background, the academic circles have a universal view the specific neurons are highly related to navigation. However, there are still many problems to be solved. For example, how do the place cells handle the information originated from the grid cells with others? How a cognitive map generates in our brain, aiding us in navigation? To mimic the mammal's spatial cognizing ability in the robots still have great challenges.

\section{ACKNOWLEDGMENT}

This work was partial supported by National Natural Science Foundation of China (No.61573029) and Beijing Natural Science Foundation (No.4162012). Thanks to the assistance of the research members in the Artificial Intelligence and Robotics Lab in Beijing University of Technology.

\section{REFERENCES}

[1] O'Keefe, J, and J. Dostrovsky. "The hippocampus as a spatial map. Preliminary evidence from unit activity in the freely-moving rat." Brain Research 34.1(1971):171-175.

[2] Jr, Butera $\mathrm{Rj}$, et al. "Analysis of the effects of modulatory agents on a modeled bursting neuron: dynamic interactions between voltage and calcium dependent systems. " Journal of Computational Neuroscience 2.1(1995):19-44.

[3] Hafting, Torkel, et al. "Microstructure of a spatial map in the entorhinal cortex." Nature 436.7052(2005):801.

[4] Blair, H. T., A. Wu, and J. Cong. "Oscillatory neurocomputing with ring attractors: a network architecture for mapping locations in space onto patterns of neural synchrony. " Philosophical Transactions of the Royal Society of London 369.1635(2013):20120526.

[5] Derdikman, Dori, and J. J. Knierim. "Introduction: A Neural Systems Approach to Space, Time, and Memory in the Hippocampal Formation." (2014):1-23.

[6] Burak, Yoram, and I. R. Fiete. "AccuratePath Integration in Continuous Attractor Network Models of, Grid Cells." Plos Computational Biology 5.2(2009):e1000291.

[7] Hasselmo, Michael E., L. M. Giocomo, and E. A. Zilli. "Grid cell firing may arise from interference of theta frequency membrane potential oscillations in single neurons." Hippocampus 17.12(2007):1252.
[8] Burgess, N, C. Barry, and J. O'Keefe. "An oscillatory interference model of grid cell firing." Hippocampus 17.9(2007):801.

[9] Bush, Daniel, and N. Burgess. "A Hybrid Oscillatory Interference/Continuous Attractor Network Model of Grid Cell Firing" Journal of Neuroscience the Official Journal of the Society for Neuroscience 34.14(2014):5065-79.

[10] Mcnaughton, B. L., C. A. Barnes, and J. O'Keefe. "The contributions of position, direction, and velocity to single unit activity in the hippocampus of freely-moving rats. " Experimental Brain Research 52.1(1983): 41.

[11] Kropff, Emilio, et al. "Speed cells in the medial entorhinal cortex." Nature 523.7561(2015):419-24.

[12] Burgess, C. P., and N. Burgess. "Controlling phase noise in oscillatory interference models of grid cell firing. " Journal of Neuroscience 34.18(2014):6224-6232.

[13] Barry, Caswell, and C. F. Doeller. "3D Mapping in the Brain." Science 340.6130(2013):279-280.

[14] Hafting, Torkel, et al. "Microstructure of a spatial map in the entorhinal cortex." Nature 436.7052(2005):801. 